\title{
La intensidad de innovación y la competitividad de micro y pequeñas empresas exportadores de artesanía textil
}

DOI: https://doi.org/10.21158/01208160.n89.2020.2848

Fecha de recepción: 15 de diciembre de 2019

Fecha de aprobación: 02 de diciembre de 2020

Fecha de publicación: 26 de abril de 2021

\author{
Alberto Magno Cutipa-Limache ${ }^{1}$ \\ Universidad Nacional del Altiplano \\ acutipa@unap.edu.pe
}

Fortunato Escobar-Mamani ${ }^{2}$ Universidad Nacional del Altiplano fescobar@unap.edu.pe

Manuel Anchapuri ${ }^{3}$

Universidad Nacional del Altiplano manchapuri@unap.edu.pe

Darío Valreymond-Tacora ${ }^{4}$ Universidad Nacional José María Arguedas dvalreymond@gmail.com

Cómo citar este artículo / To reference this article / Comment citer cet article / Para citar este artigo:

Cutipa-Limache, A. M.; Escobar-Mamani, F.; Anchapuri, M.; Valreymond-Tacora, D. (2020). La intensidad de innovación y la competitividad de micro y pequeñas empresas exportadores de artesanía textil. Revista Escuela de Administración de Negocios, (89), 155-176. DOI: https://doi.org/10.21158/01208160.n89.2020.2848

\section{Resumen}

La escasa capacidad innovadora y competitiva de micro y pequeñas empresas - mype - induce solo a la sobrevivencia en el mercado, dada su mínima participación en el mercado internacional. El objetivo del estudio fue determinar la relación entre la intensidad de la innovación y la competitividad de mypes exportadores de artesanía textil de la región Puno, Perú. El procedimiento metodológico fue descriptivorelacional con enfoque cuantitativo y diseño no experimental-transeccional. Se evaluaron 31 mypes exportadoras de artesanía textil de la región, representadas por sus gerentes, a quienes se les realizaron encuestas con dos cuestionarios de 20 ítems, se usó una escala tipo Likert de 1 a 4 alternativas de respuesta. Los resultados indican que existe una alta correlación directa y estadísticamente significativa en un $73 \%$ —rho-, $p=0,000$, a un nivel de confianza del $95 \%$, entre la intensidad de innovación y la competitividad.

\footnotetext{
Licenciado en Administración de la Universidad Nacional del Altiplano y Magíster en Administración y Gestión Estratégica con mención en Comercio Exterior de la Universidad José Carlos Mariátegui, Perú. ORCID: https://orcid.org/0000-0001-8584-6424

2 Ingeniero Economista de la Universidad Nacional del Altiplano, Maestro en Ciencias Sociales con mención en Estudios Étnicos de la Facultad Latinoamericana de Ciencias Sociales-FLACSO y Doctor en Ciencia, Tecnología y Medio Ambiente de la Universidad Nacional del Altiplano. ORCID: https://orcid.org/0000-0002-3922-4621

3 Licenciado en Administración, Magíster Scientiae en Contabilidad y Administración y Doctoris Scientiae en Contabilidad y Administración. ORCID: https://orcid.org/0000-0002-0339-2631

4 Licenciado en Administración, Magíster Scientiae en Educación con mención en Didáctica de la Educación Superior y Doctoris Scientiae en Contabilidad y Administración por la Universidad Nacional del Altiplano. ORCID: https://orcid.org/0000-00032355-0008
} 
La correlación existente implica que, a mayor intensidad de innovación en producto, en mercados, en procesos y en modelo de negocio, es mayor la competitividad en calidad de producto, al igual que la productividad y el desempeño en el mercado de las mypes exportadoras del sector de artesanía textil. Se concluye que en tanto mejore la innovación, se impactará directamente la mejora de la competitividad con miras a ampliar los horizontes de mercado internacional, lo que debe traducirse en el desarrollo de la economía; además de ser compatible con las políticas públicas de crecimiento económico equitativo, competitivo y sostenible del Gobierno del Perú.

Palabras clave: innovación empresarial: intensidad de innovación; capacidad innovadora; capacidad competitiva; competitividad empresarial; productividad; desempeño en mercado internacional.

\title{
Innovation intensity and competitiveness of micro and small textile handicraft exporting enterprises
}

\begin{abstract}
The low innovative and competitive capacity of micro and small enterprises (MSE) leads only to their survival in the market, given their minimal participation in the international market. The aim of this study was to determine the relationship between innovation intensity and competitiveness of textile handicraft exporting MSEs in the region of Puno, Peru. The methodological procedure was descriptive-relational with a quantitative approach and a non-experimental cross-sectional design. Thirty-one textile handicraft exporting MSEs in the region were evaluated, represented by their managers, who were surveyed with two 20-item questionnaires, using a Likert type scale of 1-4 response alternatives. The results show that there is a high direct and statistically significant correlation of $73 \%$-rho-, $\mathrm{p}=0.000$, at a trust level of $95 \%$, between innovation intensity and competitiveness. The existing correlation implies that the greater the intensity of innovation in product, markets, processes, and business model, the greater the competitiveness in product quality, as well as the productivity and market performance of the exporting MSEs in the textile handicraft sector. We may conclude that as long as innovation improves, it will have a direct impact on improving competitiveness to broaden international market horizons, which should translate into economic development; besides being compatible with the Peruvian government's public policies for fair, competitive, and sustainable economic growth.
\end{abstract}

Keywords: business innovation: innovation intensity; innovative capacity; competitive capacity; business competitiveness; productivity; international market performance.

\section{A intensidade da inovação e a competitividade das micro e pequenas empresas exportadoras de artesanato têxtil}

\section{Resumo}

A escassa capacidade inovadora e competitiva das micro e pequenas empresas - MPE — induz apenas à sobrevivência no mercado, dada sua mínima participação no mercado internacional. O objetivo do estudo foi determinar a relação entre a intensidade da inovação e a competitividade das MPE exportadoras de 
artesanato têxtil da região de Puno, Peru. O procedimento metodológico foi descritivo-relacional com abordagem quantitativa e desenho não experimental-transversal. Foram avaliadas $31 \mathrm{MPE}$ exportadoras de artesanato têxtil da região, representadas por seus gerentes, das quais realizaram enquetes com dois questionários de 20 itens, através da escala Likert, de 1 a 4 alternativas de resposta. Os resultados indicam que existe uma alta correlação direta e estatisticamente significativa de $73 \%$-rho-, p =0,000, a um nível de confiança de $95 \%$, entre a intensidade da inovação e a competitividade. A correlação existente implica que, quanto maior a intensidade de inovação no produto, nos mercados, nos processos e no modelo de negócio, maior será a competitividade na qualidade do produto, bem como a produtividade e o desempenho no mercado das MPE exportadoras do setor de artesanato têxtil. Conclui-se que à medida que a inovação avança, a melhoria da competitividade será diretamente impactada com vista a ampliar os horizontes do mercado internacional, o que deverá ser traduzido no desenvolvimento da economia, além de ser compatível com as políticas públicas de crescimento econômico equitativo, competitivo e sustentável do Governo do Peru.

Palavras-chave: inovação empresarial; intensidade da inovação; capacidade inovadora; capacidade competitiva; competitividade empresarial; produtividade; desempenho no mercado internacional.

\section{Relation entre innovation et compétitivité des petites entreprises exportatrices d'artisanat textile}

\section{Résumé}

La faible capacité innovatrice et compétitive des petites entreprises n'implique que leur survie sur leur marché local étant donné leur participation marginale sur les marchés internationaux. L'objectif de cette étude consiste à déterminer la relation entre l'intensité de l'innovation et la compétitivité des micro entreprises exportatrices d'artisanat textile de la région de Puno, au Pérou. La méthodologie utilisée est de type descriptif-relationnel, d'approche quantitative, et de conception non expérimentale-transactionnelle. Trente et une micro entreprises exportatrices d'artisanat textile de cette région, représentés par leurs dirigeants, ont été interrogées et évaluées au travers de deux questionnaires de 20 items et d'une échelle de type Likert de 1 à 4 réponses alternatives. Les résultats indiquent qu'il existe une forte corrélation directe, statistiquement significative de $73 \%$ - rho-, p = 0,000, d'un niveau de confiance de 95\%, entre l'intensité de l'innovation et la compétitivité. La corrélation existante implique que plus l'intensité d'innovation est importante sur le produit, les marchés, les processus et le modèle d'entreprise, plus la compétitivité en termes de qualité, de productivité et de performance sur le marché des exportateurs types du secteur de l'artisanat textile est grande. En conclusion, lorsque l'innovation s'intensifie, la compétitivité progresse, permettant à l'entreprise d'appréhender le marché international, d'améliorer son développement économique, et d'être compatible avec les politiques publiques de croissance économique équitable, compétitive et durable mise en place par le gouvernement péruvien.

Mots-clés: innovation commerciale; intensité de l'innovation; capacité d'innovation; capacité concurrentielle; compétitivité des entreprises; productivité; performance sur le marché international. 


\section{Introducción}

$\mathrm{C}$ ada día, en todas partes del mundo, se abren y cierran actividades comerciales, particularmente de las micro y pequeñas empresas - mypes - , producto de una mayor fragmentación de mercados, en los que los compradores son cada vez más exigentes, a pesar de las iniciativas de soluciones innovadoras que ofrecen las mypes. En esta categoría recae la mayor concentración de las empresas que buscan aumentar la intensidad de la innovación a fin de escapar de la competencia (Cornett, Erhemjamts y Tehranian, 2019; Salunke, Weerawardena y McColl-Kennedy, 2019).

Las cifras de fracaso y escapatoria de una buena parte de las mypes son abrumadoras en cualquier país que se analice. Particularmente, en Puno, Perú, fracasan antes de cumplir los tres años de funcionamiento. Las razones del fracaso serían la escasa «capacidad de gestión» de sus responsables, tanto en su etapa de operaciones como en la comercialización de los bienes o servicios que ofertan. Esto tiene sus incidencias en la escasa innovación frente a la competencia, lo que disminuye sus utilidades (Ghosh, Kato y Morita, 2017).

La escasa innovación disminuye la competitividad, a medida que se intensifica la competencia de toda índole y compromete a las escasas fuentes de empleo, por lo que es necesario revertir los incentivos de las mypes para la innovación incremental. No obstante, la innovación puede aplicarse en cualquier etapa del proceso, aun cuando pueden resultar en el fracaso de proyectos desarrollistas (Ghosh et al., 2017; Guevara, 2006).

En el Perú las mypes representan el 99,1 \% de todo el universo de empresas, de los cuales el 3,9 \% son de la región de Puno, la cual dinamiza la economía regional (Instituto Nacional de Estadística e Informática, 2016). Sin embargo, entre todas las actividades productivas de la región
Puno, la artesanía textil representa el $84 \%$, y de esta, solo el $6 \%$ exporta a diferentes mercados internacionales (Ministerio de Comercio Exterior y Turismo, 2015). Además, el Centro de Estudios Estratégicos-IPAE (2011) sostiene que no se refleja ningún dato relevante de la innovación de las microempresas, mientras que en las pequeñas empresas tienen una mínima participación en la intensidad de innovación de productos, procesos, factores organizacionales y comercialización. De la misma forma, el Instituto Nacional de Estadística e Informática (2015) resalta la escasa innovación de las mypes.

No obstante, un grupo de mypes objeto de estudio de artesanía textil de la región de Puno se ha mantenido en el tiempo. Este estudio examinó tres dimensiones —calidad, productividad y desempeño en el mercado- de la competitividad de dichas empresas empoderadas en su diversidad cultural y económica, si se considera que las microempresas han recibido poca atención gubernamental, la escasa participación de la academia en cuanto a su cooperación vertical clientes y proveedores-, así como la innovación para determinar los costos y beneficios empresariales.

Estos procesos presentan las mismas tendencias de otras economías emergentes que se esfuerzan en tomar decisiones y establecer un precio de venta que permita competir en el mercado con su competencia (Arellano-Cepeda et al., 2017; Shafi, Yang, Khan y Yu, 2019).

Por lo descrito, el objetivo fue determinar la relación entre la intensidad de la innovación y la competitividad de mypes exportadoras de artesanía textil de la región Puno, situada a las orillas del lago Titicaca, entre Perú y Bolivia.

El estudio busca contribuir a la literatura existente desde la postura empírica de la aplicación de la teoría y los conceptos de innovación y competitividad, lo que repercute en el desempeño 
de las empresas y su relación entre la innovación y la competitividad. De igual modo, permitirá al gobierno local, regional y nacional, así como a otros espacios, implementar políticas de cultura innovadora, fomentar la competitividad de las empresas con el fin de contribuir al desarrollo y la calidad de vida de todos los actores involucrados, todo lo cual se encuentra establecido en los Objetivos de Desarrollo Sostenible y la Agenda 2030 (Girón, 2016).
El estudio está estructurado por la revisión de la literatura que sustenta la innovación y la competitividad, luego se explica la metodología que describe la estrategia de investigación, y, por último, se presentan los resultados que permiten arribar a las conclusiones más importantes, las limitaciones y las futuras líneas de investigación.

\section{Revisión de la literatura}

\subsection{La innovación}

$S^{c}$ considera a la innovación como el elemento clave de crecimiento de las empresas, para lo cual se invierte en esto más que los competidores. De esta manera, pueden aumentar las posibilidades de éxito de manera extraordinaria o se pueden perder recursos más importantes. Las empresas con mayor intensidad de innovación tienen un impacto favorable en el crecimiento, de modo que esto se convierte en una ventaja competitiva sostenible para ofrecer nuevas soluciones de valor agregado en el tiempo (Rodeiro-Pazos et al., 2018; Salunke et al., 2019).

Así mismo, aquellas empresas que optimicen los procesos innovadores según las necesidades del mercado serán importantes en este siglo. Esto implica que tienen un alto crecimiento económico, crean demanda de productos innovadores, crean entornos comerciales hacia las innovaciones, establecen nuevos requisitos en la fuerza laboral, crean nuevos empleos y beneficios económicos, cambian las expectativas de los clientes, contribuyen al cambio de la filosofía empresarial y de la sociedad, en general, hacia un crecimiento sostenible. Es decir, se enfocan de manera integral presionando a toda la cadena de valor en la economía hacia la aceptación de innovaciones (Benešová, Kubičková, Michálková y Krošláková, 2018).
Las actividades innovadoras de los artesanos están dirigidas, básicamente, en el proceso productivo, a través de mejoras en el diseño y en función de las necesidades del mercado, el uso de nuevos materiales y la adecuación de procesos productivos con la adquisición de maquinarias y equipos, con lo cual se evidencia la mejora y el perfeccionamiento en la línea productos. Además, es un riesgo que las microempresas artesanales asuman ingresar a nuevos nichos de mercado para mantener su competitividad (Duarte y Uribe, 2012).

En un estudio de Dziallas y Blind (2019) se considera la innovación en dos etapas. En la primera - ex ante-, se relaciona el número de ideas, el porcentaje de ideas con potencial de comercialización, la dependencia de otros productos y la orientación al cliente. En la segunda —ex post—, el número de nuevos productos, la ventaja del producto, la tasa de éxito de los nuevos productos y el intervalo de tiempo entre la necesidad identificada del cliente y el lanzamiento del producto final; se identifican para la evaluación del producto y el proceso después de la introducción en el mercado.

Bajo la perspectiva planteada, la innovación viene a ser un proceso iterativo de imaginación, creatividad e invención, generador de valor para 
la organización y el mercado que, si se aplica correctamente, es una fuente de competitividad para las empresas (Martínez-Marín et al., 2009). Además, Morales y León (2013) sostienen que innovación significa poner en funcionamiento una nueva idea para crear valor.

La innovación crea valor tanto para los clientes como para la empresa (Valdés, 2009). También significa crear un valor nuevo y sustancial mediante la modificación creativa en el sistema empresarial de forma sistemática y tiene lugar en cualquiera de las dimensiones (Sawhney, Wolcott y Arroniz, 2007), es decir, innovaciones en productos, procesos, mercados y modelos de negocio (Trías y Kotler, 2011). Adicionalmente, el arte de administrar la innovación se interpreta como la autorrenovación en tecnología, en productos y modelos de negocio que se basa en principios que pueden derivarse de la ciencia de la complejidad (Lewin, Välikangas y Chen, 2017; Yu, Duan y Fan, 2020).

2.1.1 La innovación a nivel de producto, mercado, procesos y modelos de negocio.

La innovación en productos permite a las organizaciones reducir costos, crear nuevos negocios y conduce a la empresa a un desempeño superior mediante la diferenciación de productos para satisfacer con éxito las necesidades y expectativas de los clientes (Cornett et al., 2019; Jácome y Albán, 2017). Además, la mayoría de las empresas se centran en la innovación de productos significativamente mejorados y bienes nuevos (Akıncı y Utlu, 2015; Tavera, 2014), de acuerdo con las necesidades y tareas específicas de los clientes (Benešová et al., 2018).

Lainnovación deproductoserefierealaintroducción de nuevos bienes y servicios o significativamente mejorados en sus características, con nuevos usos al que se destina, es decir, mejora significativa de las características técnicas, componentes y materiales, la información integrada, la facilidad de uso u otras características funcionales (García-Pérez, GálvezAlbarracín y Maldonado-Guzmán, 2016; ValenciaDurand y Duche-Pérez, 2019) que adicionan valor al producto (Rajapathirana y Hui, 2018).
Por otra parte, Karabulut (2015) considera el desarrollo de un nuevo modelo de producto con nuevos materiales que son patentados y la mejora de productos existentes del sector que se introducen al mercado como nuevos. En suma, la innovación en productos es cualquier tipo de operación que provoque la fabricación de productos nuevos o mejorados (Tohidi y Jabbari, 2012). Además, en la micro, pequeña y mediana empresa la innovación de producto genera un mayor impacto en la mejora de su calidad, el incremento de la productividad y la cuota de mercado, entre otros (Gálvez y García, 2012).

La innovación en mercado la estudian diversos autores como innovación de mercadotecnia, mercadeo o marketing, y está referida al desarrollo de las innovaciones no tecnológicas para la comercialización (Tavera, 2014), tales como los cambios en el empaque, el diseño, el precio de un producto, sus nuevos usos, nuevos métodos de promoción, uso de sitio web de la empresa, entre otros. Es decir, es la aplicación de diferentes métodos nuevos de marketing (Karabulut, 2015), nuevos medios, nuevas técnicas de comunicación, nuevos canales de ventas (Rajapathirana y Hui, 2018), y nuevos métodos de comercialización que impliquen cambios significativos en su posicionamiento (Valencia-Durand y DuchePérez, 2019).

Así mismo, implica mercadeo social, mercadeo directo por correo electrónico, comunicación multicanal con clientes, mercadeo experiencial mediante eventos, conferencias, seminarios y talleres centrados en el segmento de clientes objetivo (Benešová et al., 2018). Además, no requieren de horizontes de investigación más amplios y conocimientos complejos (CoronadoMedina, Echeverri y Arias-Pérez, 2015). Lo que significa que, la utilización de las tecnologías de la información y comunicación (TIC) en las pymes consigue incrementar la actividad innovadora e influir positivamente en su rendimiento (Maldonado, 2011). 
La innovación en procesos conlleva una mayor inversión en maquinarias, equipos, tecnologías de información y comunicación, así como los demás procesos que hacen que el empresario decida innovar en el producto para alcanzar una mejora en la competitividad (Astudillo y Briozzo, 2016; Distanont y Khongmalai, 2018). Sin embargo, a fin de que la innovación en proceso tenga sentido, es necesario que los productos se exploten comercialmente para generar un impacto positivo y superior en el mercado en relación con la competencia (Arias, Durango y Millán, 2015).

Por tanto, la innovación en procesos sucede cuando se introduce un nuevo o significativamente mejor método de trabajo, nuevas técnicas, materiales, procesos de producción, distribución o programas informáticos (Valencia-Durand y Duche-Pérez, 2019), mejoras o cambios en los procesos productivos, adquisición de nuevos equipos (García-Pérez et al., 2016), cambios en los métodos de fabricación, control de costos en los procesos y registros de tiempo, desde los materiales hasta la entrega de productos; (Karabulut, 2015), aumento en la velocidad de implementación, cambios en el edificio que opera de forma plana, procesos interactivos en línea y métodos que permiten la instrucción laboral, entre otros (Rajapathirana y Hui, 2018). En suma, se refiere a los procesos significativamente mejorados y a los procesos nuevos implementados por las empresas (Tavera, 2014).

La innovación en modelo de negocio surge por las innovaciones en productos, procesos, a nivel organizacional y de mercado, con base en el know how de la empresa y la ventaja otorgada por el posicionamiento de la marca en el mercado (Gonzales y Gonzáles, 2017). También es necesario procesar y entender el cambio para introducir y alinear la innovación como parte de una estrategia permanente en los nuevos modelos de negocios (Álvarez-Falcón, 2017). Además, implica la modificación profunda en generar valor para la empresa, los clientes y la sociedad, y con miras a su implementación requerirá una restructuración o sustitución de los modelos obsoletos, principalmente, en nueve módulos que impactan de forma distinta en la empresa y a sus grupos de interés (Trías y Kotler, 2011; Osterwalder y Pigneur, 2011).

Finalmente, intensificar los esfuerzos de innovación permite mejorar la competitividad en un entorno cada vez más exigente, con tendencias de alto grado de novedad para la empresa y el mercado (Astudillo y Briozzo, 2016); puesto que la innovación está relacionada con el éxito competitivo empresarial (Valencia-Durand y Duche-Pérez, 2019). Lo anterior implica que las mypes pueden integrar, aprender y reconfigurar sus recursos internos y externos con rapidez y eficacia, para así adaptarse fácilmente al entorno competitivo de constante cambio y mejorar el rendimiento (Khan, Xuehe, Atlas y Khan, 2018).

Además, resulta necesario mencionar que la actividad innovadora en áreas propicias aumenta el nivel de desarrollo de la infraestructura, mejora la calidad del trabajo y el nivel de productividad laboral, en suma, mejora la competitividad (Bezpalov, Fedyunin, Solopova, Avtonomova y Lochan, 2019).

\subsection{La competitividad}

En el Perú, desde la década del 2000, las políticas de fomento a las mypes se incrementaron gracias al Plan Nacional de Promoción y Formalización para la Competitividad y Desarrollo de la Micro y Pequeña Empresa, de acuerdo con la Ley 28015 de 2003, la cual persigue, entre otros objetivos, promover la cooperación empresarial, mejorar la calidad del empleo, facilitar la formalización de las mypes y aumentar su productividad y rentabilidad. Estas propuestas están acompañadas por el apoyo a los programas de I + D y el Plan Nacional de Ciencia, Tecnología e Innovación para la Competitividad y el Desarrollo Humano (Heredia-Rodríguez y Sánchez-Macías, 2016). 
Bajo el escenario planteado, existen diversos elementos que explican la competitividad empresarial, tales como la tecnología, la eficiencia en la producción, la orientación al mercado, los ingresos, la relación entre precio-costo, la tasa de ganancia, la rentabilidad, la tasa de retorno, la participación en el mercado, la innovación, el capital intelectual, el conocimiento del mercado, la investigación y el desarrollo, la asociatividad, las estrategias empresariales, la diferenciación, la gestión financiera, la cultura organizacional y los servicios, entre otros, que son imprescindibles a fin de medir la competitividad (Porter, 1990; Cabrera, López y Ramírez, 2011; Pat, Caamal y Ávila, 2009). Además, Camacho, Hernández y Mayorga (2016) manifiestan que existe una correlación positiva entre la innovación y la competitividad, lo que implica que, si las empresas siguen implementando y mejorando la intensidad de la innovación, lograrán altos niveles de competitividad.

\subsubsection{La competitividad basada en calidad, productividad y desempeño en el mercado.}

La competitividad es la capacidad que tiene una empresa para logar una posición notoria en el mercado. La capacidad se entiende como la forma de combinar y aprovechar los recursos existentes en la empresa (Becerra y García, 2008). Además, se basa en las habilidades, el conocimiento tecnológico y la experiencia que emplean las personas en las diferentes funciones que desarrolla la empresa (Hitt, Ireland y Hoskisson, 2015).

Entre estas capacidades, resalta el aprovechamiento de la tecnología existente que apoye la innovación incremental, es decir, la mejora continua con las actividades innovadoras que apuntan al uso de una tecnología completamente nueva —innovación discreta- La innovación incremental a menudo tiene una relevancia limitada para la nueva tecnología inventada por una innovación discreta exitosa (Crittenden, Crittenden y Ajjan, 2019; Ghosh et al., 2017).
Ahora bien, en cuanto a la competitividad y la calidad, la competitividad es la capacidad de producir y mercadear productos a mejor precio, calidad y oportunidad (Porter, 1990), generar productos con valor agregado, basándose en los elementos de costos, beneficios, características (Münch, 2007) y excelencia, al ofrecer lo mejor y considerar distintos factores para ganarse a los clientes (Yarce, 2000), crear valor y suministrar servicios de acuerdo con las expectativas (Mendoza (2010), así como rivalizar con otras empresas a fin de lograr una posición competitiva favorable y obtener un desempeño superior (Aragón y Rubio (2005). Además, cabe resaltar que, una empresa es más competitiva respecto a otra cuando produce los mismos productos, pero de mejor calidad, haciendo uso adecuado de los recursos disponibles, y sale al mercado internacional a competir y a ganar (D'Alessio, 2015).

Por otro lado, una mayor percepción de calidad de la marca mejorará la satisfacción de los usuarios en la compra de productos, aspecto que favorece las ventas (Arroyo-Cañada, Sánchez-Torres y Solé-Moro, 2017). Otro elemento indispensable en la forma de gestionar las organizaciones, así como la forma en que deben de conducirse todas las personas que colaboran en ella, de modo que representa el cumplimiento legal el principal elemento en su comportamiento ético, seguido de la relación equitativa precio-calidad de producto o servicio (Ríos-Manríquez, 2017).

En cuanto a la competitividad, la productividad y el desempeño en el mercado, las organizaciones han de promover buenas relaciones laborales, motivar a sus trabajadores y retroalimentar el desempeño si quieren incrementar la productividad del trabajador en el desarrollo de las actividades laborales (Suarez, Rodríguez y Muñoz, 2017). Por otra parte, los bajos niveles de aseguramiento de la calidad como certificado de calidad en procesos y certificado de calidad de producto en las empresas (Franco-Ángel y Urbano, 2019) repercuten de forma negativa en el desempeño del mercado. 
En este caso, el factor calidad de producto a precios competitivos será un elemento que las organizaciones deben tener como prioridad en el diseño de estrategias de producción y comercialización, aunque la literatura del marketing de nichos sugiere que, en este tipo de mercado, el valor añadido reposa en la variable de diferenciación del producto o rareza y no en el precio (OrengoSerra y Ortíz-Soto, 2017).

Por último, la competitividad empresarial se puede alcanzar a través del uso adecuado y diferenciado de recursos naturales, financieros y tecnológicos, utilizados de una manera superior que la competencia. También se crea y se logra a través de un largo proceso de aprendizaje, negociación, liderazgo y por medio de los grupos de interés que configuran la dinámica de conducta organizativa, tales como los accionistas, los directivos, los empleados, los acreedores, los clientes, los competidores, el mercado, el gobierno y la sociedad en general (Ascón-Villa et al., 2018).

\section{Metodología}

$\mathrm{E}$ estudio fue realizado en la región de Puno, entre los meses de junio a diciembre del 2016, en el sureste de Perú, a orillas del lago Titicaca, a 3810 metros sobre el nivel del mar, $13^{\circ} 0066^{\prime} 00^{\prime \prime}$ y $17^{\circ} 17^{\prime} 30^{\prime \prime}$ de latitud sur y los $71^{\circ} 06^{\prime} 57^{\prime \prime}$ y $68^{\circ} 48^{\prime} 46^{\prime \prime}$ de longitud oeste del meridiano de Greenwich.

El procedimiento metodológico fue descriptivorelacional con enfoque cuantitativo y diseño no experimental-transeccional. La población objeto de estudio fueron 31 mypes exportadoras de artesanía textil, registradas en el directorio de empresas exportadores de la Dirección Regional de Comercio Exterior y Turismo en la región de Puno. Teniendo en cuenta que, según Córdova (2013), cuando la población es pequeña, es recomendable realizar el estudio en toda la población, se encuestó a los gerentes de la totalidad de mypes exportadoras del sector de artesanía textil. Para esto, los instrumentos de recolección de datos fueron dos cuestionarios: uno para la variable intensidad de innovación y el otro dirigido a la variable competitividad, estos fueron sometidos a la prueba de validez y confiabilidad para asegurar su eficacia en la recopilación de datos.

En la variable de intensidad de innovación se consideraron cuatro dimensiones: innovación en productos, innovación en mercados, innovación en procesos e innovación en modelo de negocio. Además, en la variable competitividad se definieron tres dimensiones: calidad de producto, productividad y desempeño en el mercado. También para cada una de las variables se formuló un total de 20 ítems, considerando como alternativas de respuestas una escala 1 a 4 puntos, donde: $1=$ nunca, $2=$ pocas veces, $3=$ casi siempre y $4=$ siempre (véanse las tablas 1 y 2 ). 
Tabla 1. Variable intensidad de innovación, dimensiones e ítems

\section{Innovación en productos}

1. Mi empresa ha desarrollado nuevos productos para el mercado internacional entre los años 2014 al 2016.

2. Mi empresa se ha esforzado en incrementar cantidad de desarrollo de nuevos productos entre los años 2014 a 2016.

3. Los productos introducidos al mercado fueron novedosos tanto para la empresa como para el mercado entre los años 2014 a 2016.

4. Mi empresa se ha esforzado en asignar una marca a sus productos nuevos y registrar en Indecopi entre los años 2014 a 2016.

5. Mi empresa ha modificado y mejorado permanentemente los productos existentes entre los años 2014 a 2016.

Innovación en mercados

6. Mi empresa intensificó el uso las TIC en la comercialización de sus productos —correo electrónico, búsqueda de información en internet, redes sociales - entre los años 2014 a 2016.

7. Mi empresa promocionó sus productos a través de una tienda virtual a mercados internacionales entre los años 2014 a 2016.

8. Mi empresa ha realizado cambios y mejoras significativas del envase, empaque y embalaje de los productos a exportar entre los años 2014 y 2016.

9. Mi empresa ha intensificado el uso de nuevos canales de comercialización como el Serpost, Courier, DHL, Exporta Fácil y otros entre los años 2014 y 2016.

Innovación en procesos

10. Mi empresa implementó nuevos o mejores métodos de trabajo en el proceso de producción de artesanía textil entre los años 2014 y 2016.

11. Mi empresa renovó sus equipos, herramientas y máquinas de tejer en el área de operaciones entre los años 2014 y 2016.

12. Mi empresa ha introducido nuevos o mejores métodos de administración de procesos entre los años 2014 y 2016.

\section{Innovación en modelo de negocio}

13. Mi empresa externalizó algunas actividades a terceros — socios o aliados claves — para cumplir con la meta de producción entre los años 2014 y 2016.

14. Mi empresa se esforzó en buscar alianzas con otras empresas o instituciones entre los años 2014 a 2016.

15. Mi empresa buscó proactivamente la adecuación y adaptación a los cambios del entorno competitivo entre los años 2014 a 2016.

16. Mi empresa realizó cambios y mejoras en la estructura organizativa de acuerdo con las exigencias del entorno competitivo entre los años 2014 a 2016.

17. Mi empresa se esforzó en mantener relaciones duraderas con sus clientes mediante plataforma virtual, Facebook, Twitter, LinkedIn, YouTube, etc., entre los años 2014 a 2016.

18. La oferta de mis productos fue única y diferente en diseño, precio, velocidad de entrega y servicio, aspectos que sorprendieron a nuestros clientes entre los años 2014 a 2016.

19. Los productos que se ofrecieron en mi empresa fueron adaptados a las necesidades específicas de cada mercado internacional entre los años 2014 a 2016.

20. Mi empresa realizó permanentemente nuevos diseños de productos como propuesta de valor para el mercado internacional entre los años 2014 a 2016.

Fuente. Elaboración propia. 
Tabla 2. Variable competitividad, dimensiones e ítems

\begin{tabular}{|l|}
\hline Calidad del producto \\
\hline 1. Mi empresa se esfuerza en estandarizar sus productos de acuerdo con el mercado internacional. \\
2. Mi empresa realiza controles de calidad de los artículos de artesanía textil según ficha técnica. \\
3. Mi empresa promueve la obtención de certificado de calidad para exportar. \\
4. Mi empresa promueve la implementación de norma de calidad para la exportación de sus productos. \\
5. Los productos de mi empresa se diferencian claramente de los competidores. \\
\hline Productividad \\
\hline 6. En mi empresa se busca incrementar la línea de productos para exportar mediante la innovación. \\
7. En mi empresa se busca diversificar nuevas líneas de productos por medio de la innovación. \\
8. Mi empresa se esfuerza en incrementar el volumen de producción mediante la innovación. \\
9. En mi empresa el incremento del volumen de producción es significativo por las innovaciones realizadas. \\
10. Mi empresa posee una capacidad de producción superior a los competidores por las innovaciones realizadas. \\
11. La capacidad de producción de mi empresa ha mejorado significativamente por las innovaciones realizadas. \\
12. Mi empresa ha mejorado en el uso de los recursos - materiales y tiempo- por las innovaciones realizadas. \\
13. En mi empresa se han reducido los costos en todos los niveles por las innovaciones realizadas. \\
14. La reducción de los costos en mi empresa es significativa por las innovaciones realizadas. \\
\hline Desempeño en el mercado \\
\hline 15. Mi empresa se esfuerza en incrementar el número de clientes internacionales por medio de la innovación. \\
16. El incremento de nuevos clientes de mi empresa es significativo por las innovaciones realizadas. \\
17. Mi empresa se esfuerza en incrementar las ventas de sus productos mediante la exportación. \\
18. El incremento en las ventas de productos exportados es significativo por las innovaciones realizadas. \\
19. Mi empresa se esfuerza en ingresar a nuevos mercados internacionales con sus productos de artesanía textil. \\
20. Mi empresa consigue cada año al menos un nuevo mercado internacional con las innovaciones realizadas. \\
\hline
\end{tabular}

Fuente. Elaboración propia.

\subsection{Validez y confiabilidad del instrumento}

La validez de los instrumentos se desarrolló mediante juicio de expertos, considerando los criterios de claridad, objetividad, actualidad, organización, coherencia estructural, coherencia semántica, consistencia teórica, metodología, estructura formal y originalidad. Se tuvo en cuenta una escala de valoración de 0,0 a 2,0 donde: $0,0=$ muy deficiente, $0,5=$ deficiente,
$1,0=$ regular, $1,6=$ bueno y $2,0=$ muy bueno - . Por tanto, los resultados (véase la Tabla 3 ) de la evaluación de expertos para el nivel porcentual es de $90,5 \%-905 / 10=90,5-905 / 10=90.5$ para la variable intensidad de innovación y $92,5 \%$ $-925 / 10=92,5-925 / 10=92.5$ para la variable competitividad. En definitiva, ambas variables obtienen un valor superior al $75 \%$, de modo que valida los dos instrumentos de recolección de datos.

Tabla 3. Validez del instrumento de intensidad de innovación y competitividad

\begin{tabular}{|c|c|c|c|c|c|c|c|c|c|c|c|c|c|c|}
\hline \multirow[t]{2}{*}{ Criterios de evaluación } & \multicolumn{7}{|c|}{$\begin{array}{l}\text { Número de expertos para intensidad } \\
\text { de innovación }\end{array}$} & \multicolumn{7}{|c|}{$\begin{array}{l}\text { Número de expertos para } \\
\text { competitividad }\end{array}$} \\
\hline & E1 & E2 & E3 & E4 & E5 & $\Sigma$ & $\%$ & E1 & $\mathrm{E} 2$ & E3 & E4 & E5 & $\Sigma$ & $\%$ \\
\hline Claridad & 2 & 1,5 & 1 & 2 & 2 & 8.5 & 85 & 2 & 2 & 2 & 2 & 2 & 9,5 & 95 \\
\hline Objetividad & 1,5 & 2 & 1,5 & 2 & 2 & 9 & 90 & 2 & 2 & 2 & 2 & 2 & 9,5 & 95 \\
\hline Actualidad & 1,5 & 1,5 & 1,5 & 2 & 2 & 8,5 & 85 & 2 & 2 & 2 & 2 & 2 & 10 & 100 \\
\hline Organización & 2 & 1,5 & 1,5 & 1,5 & 2 & 8,5 & 85 & 2 & 1,5 & 2 & 2 & 2 & 9 & 90 \\
\hline Coherencia estructural & 2 & 2 & 1,5 & 2 & 2 & 9,5 & 95 & 1,5 & 2 & 2 & 1,5 & 2 & 8,5 & 85 \\
\hline Coherencia semántica & 2 & 1,5 & 1,5 & 2 & 2 & 9 & 90 & 2 & 1,5 & 2 & 2 & 2 & 9 & 90 \\
\hline Consistencia teórica & 2 & 2 & 1,5 & 2 & 2 & 9,5 & 95 & 2 & 1,5 & 2 & 2 & 2 & 9 & 90 \\
\hline
\end{tabular}




\begin{tabular}{|c|c|c|c|c|c|c|c|c|c|c|c|c|c|c|}
\hline Metodología & 2 & 2 & 1,5 & 1,5 & 2 & 9 & 90 & 1,5 & 2 & 2 & 2 & 2 & 9 & 90 \\
\hline Estructura formal & 2 & 2 & 1,5 & 2 & 2 & 9,5 & 95 & 2 & 2 & 2 & 2 & 2 & 9,5 & 95 \\
\hline Originalidad & 2 & 2 & 1,5 & 2 & 2 & 9,5 & 95 & 2 & 2 & 2 & 2 & 2 & 9,5 & 95 \\
\hline Total & 905 & Total & 925 & & & & & & & & & & & \\
\hline
\end{tabular}

Nota. E1 = Experto 1, E2 = Experto 2, E3 = Experto 3, E4 = Experto 4 y E5 = Experto 5

Fuente. Elaboración propia.

La confiabilidad de los instrumentos para las variables de intensidad de innovación y competitividad se calculó mediante el coeficiente de alfa de Cronbach - $-\alpha$-, para lo cual se utilizó el programa estadístico SPSS y se tuvo como fundamento la siguiente fórmula: $\alpha=(\mathrm{k} / \mathrm{k}-1)\left[1-\left(\sum\right.\right.$ $\left.\left.S_{r}{ }^{2} / S_{1}{ }^{2}\right)\right]$; donde $k=$ número de reactivos en la escala, $\mathrm{S}_{\mathrm{r}}^{2}=$ varianza de cada reactivo y $\mathrm{S}_{1}{ }^{2}=$ varianza del instrumento. Por consiguiente, los resultados (véase la Tabla 4) evidencian que el coeficiente de alfa de Cronbach es de $86 \%$ para la variable intensidad de innovación y $84 \%$ para la variable competitividad, con 20 ítems cada variable, lo que significa que los instrumentos son altamente confiables (Córdova, 2013).

Tabla 4. Estadísticos de fiabilidad

\begin{tabular}{|c|c|c|c|}
\hline \multicolumn{2}{|c|}{ Intensidad de innovación } & \multicolumn{2}{c|}{ Competitividad } \\
\hline Alfa de Cronbach & N de elementos & Alfa de Cronbach & N de elementos \\
\hline 0,86 & 20 & 0,84 & 20 \\
\hline
\end{tabular}

Fuente. Elaboración propia.

\subsection{Valoración de resultados}

A fin de interpretar los resultados se calculó el valor total de la variable intensidad de innovación con 80 puntos, las dimensiones de innovación en productos 20 puntos, innovación en mercados 16 puntos, innovación en procesos 12 puntos e innovación en modelo de negocio 32 puntos. De la misma forma, el valor total de la variable competitividad fue de 80 puntos, las dimensiones de calidad del producto 20 puntos, productividad 36 puntos y desempeño en el mercado 24 puntos. Así mismo, se establece una escala cuantitativa de 0,00 a 1,00 y una escala de cualificación que va desde muy bajo hasta muy alto (véase la Tabla 5).

Tabla 5. Escala de valoración de variables y dimensiones

\begin{tabular}{|c|c|c|c|c|c|c|c|c|c|}
\hline \multirow{2}{*}{ Escala cuantitativa } & \multirow{2}{*}{ Escala de cualificación } & \multicolumn{7}{|c|}{ Escala cuantitativa de puntajes } \\
\cline { 3 - 10 } & & VII y VC & DIP & DIM & DIPR & DIMN & DCP & DPR & DDM \\
\hline$[0,00-0,25]$ & Muy bajo & $0-20$ & $0-5$ & $0-4$ & $0-3$ & $0-8$ & $0-5$ & $0-9$ & $0-6$ \\
\hline$[0,26-0,45]$ & Bajo & $21-36$ & $6-9$ & $5-7$ & $4-5$ & $9-14$ & $6-9$ & $10-16$ & $7-11$ \\
\hline$[0,46-0,65]$ & Moderado & $37-52$ & $10-13$ & $8-10$ & $6-8$ & $15-21$ & $10-13$ & $17-23$ & $12-16$ \\
\hline$[0,66-0,85]$ & Alto & $53-68$ & $14-17$ & $11-14$ & $9-10$ & $22-27$ & $14-17$ & $24-31$ & $17-20$ \\
\hline$[0,86-1,00]$ & Muy alto & $69-80$ & $18-20$ & $15-16$ & $11-12$ & $28-32$ & $80-20$ & $32-36$ & $21-24$ \\
\hline
\end{tabular}

Nota. VII = Variable intensidad de innovación, VC = Variable competitividad, DIP = Dimensión innovación en productos, DIM = Dimensión innovación en mercados, DIPR = Dimensión innovación en procesos, DIMN = Dimensión innovación en modelo de negocio, DCP = Dimensión calidad del producto, DPR = Dimensión productividad y DDM = Dimensión desempeño en el mercado.

Fuente. Adaptado de Monzón, Hernández-Pérez y Nogueira-Rivera, 2015. 


\section{Resultados}

$\mathrm{L}^{\infty}$ os resultados indican que la intensidad de innovación es moderada y alta en la mayoría de las mypes exportadores de la región Puno. La innovación en producto representa el 41,9\%, al igual que la innovación en mercados (véase la Tabla 6). Estos resultados se deben a la intensificación de las actividades de desarrollo de nuevos productos y a la mejora de los productos existentes. Así mismo, en la innovación en mercados se intensificó el uso de nuevos canales de promoción tales como las tecnologías de la información y la comunicación (TIC); también se realizaron cambios o mejoras significativas en los envases y embalajes de los productos para su comercialización. Esto implica nuevas estrategias de innovación y competitividad, hallazgos que corroboran los otros estudios (Ghosh et al., 2017; Kang, Baek y Lee, 2019).

Con respecto a la intensidad de innovación en procesos se encuentra entre bajo y muy alto en mayor proporción - $29 \%$ - Esto es producto de la implementación de nuevos equipos y máquinas de trabajo, nuevos o mejores métodos de producción y administración, principalmente. Por último, en cuanto a la innovación en el modelo de negocio, la mayoría — 48,4 \% — de las mypes exportadores de artesanía textil obtuvieron un puntaje alto, debido a la intensidad de innovación en la propuesta de valor, aliados clave y adecuación de la organización al cambio (véase la Tabla 6).

Los hallazgos de este estudio siguen la misma tendencia de los de Martínez-Marín et al. (2009), el Centro de Estudios Estratégicos-IPAE (2011) y el Instituto Nacional de Estadística e Informática (2015), especialmente en la innovación de productos, de modo que es la más visible. Sin embargo, el nivel de innovación de procesos, mercados y modelos de negocio conlleva una mayor inversión en el empresario para lograr la competitividad (Astudillo y Briozzo, 2016).

La innovación en cualquiera de sus niveles crea un valor nuevo y significativo tanto para los clientes como para la empresa mediante la modificación creativa y sistemática en sus diversas dimensiones (Sawhney et al., 2007). En definitiva, los casos estudiados son coherentes con otros estudios de gran importancia en el propósito de inducir a la formulación de políticas de innovación, incluyendo invención, modelo de utilidad y patentes de diseño de apariencia (Yu et al., 2020).

Tabla 6. Innovación según dimensiones

\begin{tabular}{|c|c|c|c|c|}
\hline Escala & $\begin{array}{c}\text { Innovación en } \\
\text { productos }\end{array}$ & $\begin{array}{c}\text { Innovación en } \\
\text { mercados }\end{array}$ & $\begin{array}{c}\text { Innovación en } \\
\text { procesos }\end{array}$ & $\begin{array}{c}\text { Innovación en } \\
\text { modelos de negocio }\end{array}$ \\
\hline Muy alto & $19,4 \%$ & $29 \%$ & $29 \%$ & $29 \%$ \\
\hline Alto & $38,7 \%$ & $19,4 \%$ & $22,6 \%$ & $48,4 \%$ \\
\hline Moderado & $41,9 \%$ & $41,9 \%$ & $9,7 \%$ & $22,6 \%$ \\
\hline Bajo & $0 \%$ & $9,7 \%$ & $29 \%$ & $0 \%$ \\
\hline Muy bajo & $0 \%$ & $0 \%$ & $9.7 \%$ & $0 \%$ \\
\hline Total & $100 \%$ & $100 \%$ & $100 \%$ & $100 \%$ \\
\hline
\end{tabular}

Fuente. Elaboración propia. 
Los resultados (véase la Tabla 7) evidencian que, el mayor porcentaje - 54,8 \% - fue de alto, y el 35,5 $\%$ moderado, en la intensidad de innovación de las mypes exportadores de artesanía textil de la región de Puno. Estos hallazgos reflejan el esfuerzo de las actividades de innovación en el desarrollo de nuevos productos, modificación o mejora permanente de los productos existentes; el uso de las TIC para promocionar productos de exportación, cambios o mejoras significativas en el envase, empaque y embalaje de los productos; nuevos canales de comercialización como el exporta fácil y courier, nuevos o mejores métodos de trabajo en el proceso de producción y en la administración que agilicen los procesos y reduzcan costos; nuevas alianzas con otras organizaciones para la producción; cambios en la estructura organizativa a fin de adaptarse al entorno competitivo; nuevas formas de mantener relaciones duraderas con los clientes y nuevas propuestas de valor como el corazón del modelo de negocio, a través de una oferta de productos únicos en diseño, precio y entrega oportuna de acuerdo con las necesidades de cada mercado internacional.

Tabla 7. Intensidad de innovación de las mypes exportadores de artesanía textil

\begin{tabular}{|c|c|c|c|}
\hline Escala & Puntaje & Frecuencia & Porcentaje \\
\hline Muy alto & {$[69-80]$} & 3 & 9,7 \\
\hline Alto & {$[53-68]$} & 17 & 54,8 \\
\hline Moderado & {$[37-52]$} & 11 & 35,5 \\
\hline Bajo & {$[21-36]$} & 0 & 0 \\
\hline Muy bajo & {$[0-20]$} & 0 & 0 \\
\hline Total & & 31 & 100 \\
\hline
\end{tabular}

Fuente. Elaboración propia.

La competitividad es la capacidad que tiene una empresa para generar valor mediante la productividad, la calidad y el buen desempeño en el mercado. En esa línea, se observa que las mypes exportadores de artesanía textil de la región Puno obtuvieron una escala de calificación alta en mayor proporción, según las dimensiones de productividad -61,3\%—, calidad de producto - 54,8 \% - y desempeño en el mercado - 38,7\% - competitivo (véase la Tabla 8).

El elemento más destacado para la competitividad es la productividad. En ese sentido, los hallazgos de este estudio concuerdan con la afirmación de D’Alessio y Del Carpio (2011); pues esta facilita la solidez, el dinamismo y la capacidad de competencia en los mercados internacionales (Correa, 2005).
De la misma manera, la calidad del producto es un requisito para lograr la competitividad. Tal es así que los exportadores de artesanía textil de la región Puno se encuentran en ese camino de lograr la estandarización y la calidad del producto de exportación.

Estos hechos reafirman las conclusiones de Paredes (2017) respecto a las empresas que exportan artesanía textil. Sin embargo, difieren con los resultados de Catacora (2013), quien manifiesta que la competitividad de las mypes se ve limitada por la calidad de sus productos. Finalmente, la dimensión de desempeño de mercado se alinea a lo establecido por Jasso (1998), quien manifiesta que las mypes en América Latina son pocas y débiles en mercados internacionales. 
Tabla 8. Competitividad según dimensiones

\begin{tabular}{|c|c|c|c|}
\hline Escala & Calidad del producto & Productividad & Desempeño en el mercado \\
\hline Muy alto & $38,7 \%$ & $3,2 \%$ & $0 \%$ \\
\hline Alto & $54,8 \%$ & $61,3 \%$ & $35,5 \%$ \\
\hline Moderado & $6,5 \%$ & $32,3 \%$ & $38,7 \%$ \\
\hline Bajo & $0 \%$ & $3,2 \%$ & $22,6 \%$ \\
\hline Muy bajo & $0 \%$ & $0 \%$ & $3,2 \%$ \\
\hline Total & $100 \%$ & $100 \%$ & $100 \%$ \\
\hline
\end{tabular}

Fuente. Elaboración propia.

La competitividad en estos tiempos de constante cambio dinámico resulta imperante en las empresas para garantizar la sostenibilidad en el mercado. Desde esta perspectiva, los resultados obtenidos (véase la Tabla 9) reflejan el nivel de competitividad que alcanzaron las mypes exportadores de artesanía textil, obteniendo el 51,6 \% un nivel moderado de competitividad y el $45,2 \%$ un nivel alto.

Estos resultados se sustentan en la estandarización alcanzada de los productos, el control de calidad mediante las fichas técnicas, la diferenciación del producto - dado que el mercado internacional exige productos con diseños exclusivos-, el crecimiento de la productividad mediante el aumento de línea de productos, el volumen de producción, la capacidad de producción, el uso adecuado de los recursos, la reducción de costos, $y$, en menor medida, por el desempeño de mercado con el incremento de clientes, el volumen de ventas $y$ el ingreso a nuevos mercados internacionales. En suma, las actividades innovadoras realizadas por las pymes exportadoras de artesanía textil son favorables para la competitividad.

Los hallazgos de este estudio van en la misma vía sustentada en la conclusión de Mora-Riapira, Vera-Colina y Melgarejo-Molina (2015), quienes consideran que las mypes con mayores niveles de competitividad tendrán la posibilidad de enfrentar a competidores e ingresar y expandir el negocio en los mercados internacionales. Además, refuerzan la literatura estudiada por Porter (1990), Yarce (2000), Aragón y Rubio (2005), D 'Alessio (2015) y Mendoza (2010). Esto significa un impacto positivo en la sobrevivencia y sostenibilidad en los mercados internacionales para las mypes exportadores del sector de artesanía textil de la región Puno.

Tabla 9. Competitividad de las mypes exportadores de artesanía textil

\begin{tabular}{|c|c|c|c|}
\hline Escala & Puntaje & Frecuencia & Porcentaje \\
\hline Muy alto & {$[69-80]$} & 1 & 3,2 \\
\hline Alto & {$[53-68]$} & 14 & 45,2 \\
\hline Moderado & {$[37-52]$} & 16 & 51,6 \\
\hline Bajo & {$[21-36]$} & 0 & 0 \\
\hline Muy bajo & {$[0-20]$} & 0 & 0 \\
\hline Total & & 31 & 100 \\
\hline
\end{tabular}

Fuente. Elaboración propia. 
Los resultados de la relación entre las variables intensidad de innovación y competitividad (véase la Tabla 10) reflejan el grado de correlación, que fue positiva y alta con un coeficiente de correlación Rho Spearman $=0,726$, valor- $\mathrm{p}=0,000$, menor a $\alpha=$ 0,05 y a un nivel de confianza del $95 \%$. Por tanto, se afirmó la hipótesis de que existe una relación entre la intensidad de innovación y la competitividad de las mypes exportadores del sector de artesanía textil de la región Puno. Además, la figura 1 del diagrama de dispersión refuerza los resultados antes expuestos, ya que se observa una relación positiva y directa, es decir, cuando aumenta el nivel de intensidad de innovación en el producto, mercados, procesos e innovación en modelo de negocio, aumenta la competitividad mediante la calidad del producto, la productividad y el desempeño en el mercado de las mypes exportadores de artesanía textil de la región Puno.
Estos hallazgos coinciden con las conclusiones de Camacho et al. (2016), quienes manifiestan que existe una correlación positiva entre la innovación y la competitividad al implementar y mejorar la intensidad de la innovación, pues lograrán altos niveles de competitividad. Así mismo, va en un sentido a las conclusiones de Moraes, Buesa, Johannes y Baumert (2018), quienes consideran que el impacto de la innovación en la competitividad es menor del que se podría esperar, ya que cambia según el sector y no necesariamente se traduce en éxito empresarial debido a las condiciones del entorno tecnológico, la estructura de los mercados y las características del sector. En suma, la relación entre la innovación y la competitividad es innegable para cualquier micro y pequeña empresa enfocada en sobrevivir en los mercados globales.

Tabla 10. Relación entre intensidad de innovación y competitividad

\begin{tabular}{|c|c|c|}
\hline \multicolumn{2}{|c|}{} & Competitividad \\
\hline \multirow{4}{*}{ Intensidad de innovación } & Coeficiente de correlación de Rho Spearman & $0,726^{* *}$ \\
\cline { 2 - 3 } & Sig. - bilateral- & 0,000 \\
\cline { 2 - 3 } & $\mathrm{N}$ & 31 \\
\hline
\end{tabular}

**. La correlación es significativa en el nivel 0,01 — bilateral-.

Fuente. Elaboración propia.

Figura 1. Diagrama de dispersión: intensidad de innovación y competitividad

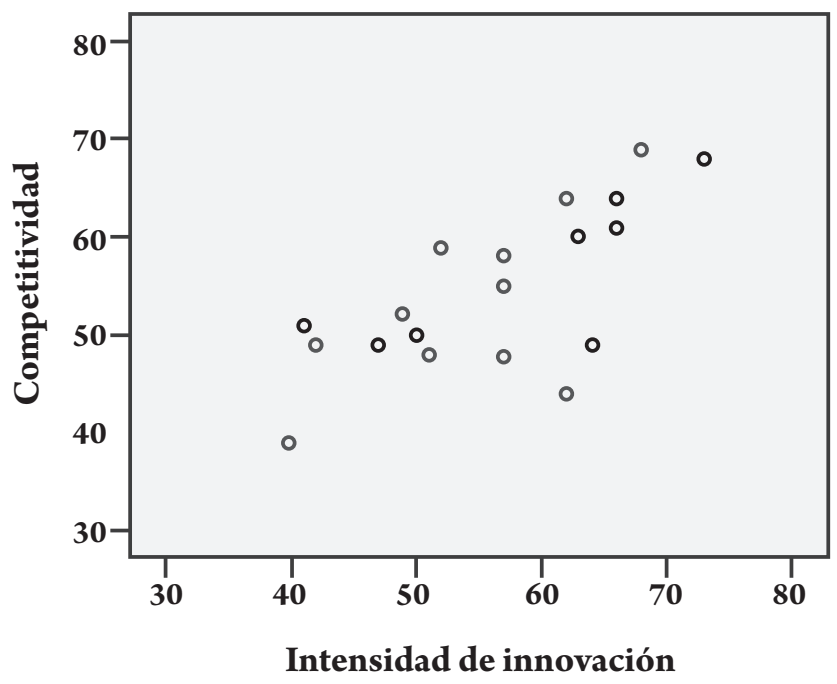

Fuente. Elaboración propia. 
Las mypes estudiadas están inmersas en mercados cada vez más inciertos y competitivos, no solo en la región de Puno y en los mercados a las que exporta sus productos; principalmente, para mercados turísticos, a menudo son presionadas para innovar en una amplia gama de tipos de innovación. El proceso de innovación y competitividad induce a la diversidad de la innovación y a la búsqueda de sinergias de capacidades dirigidas a mejorar sus productos.
Estas acciones constituyen una estrategia de cooperación entre las mypes de Puno y otras aledañas para garantizar la sostenibilidad de sus entregas. Por tanto, el desarrollo de negocios textiles se desenvuelve de acuerdo con los principios del desarrollo sostenible y la responsabilidad social que vincula los resultados sociales, financieros y ambientales, aun cuando fue limitado (Reshetnikova y Magomedov, 2020; Verreynne et al., 2019).

\section{Conclusión}

$\mathrm{E}^{1}$ estudio buscó determinar la relación entre la intensidad de innovación y la competitividad de mypes exportadoras de artesanía textil de la región Puno. En esta dirección, los resultados evidencian que existe una relación alta y significativa entre la intensidad de innovación y la competitividad en las mypes exportadores de artesanía textil de la región Puno, Perú, lo que se corrobora, además, por otras investigaciones (Camacho et al., 2016; Moraes et al., 2018). Por tanto, implica que a mayor intensidad de innovación en productos, mercados, procesos y modelo de negocio, mayor será la competitividad con base en la calidad de producto, la productividad y el desempeño en el mercado internacional de las mypes exportadoras del sector.

Sin embargo, se requiere seguir impulsando de manera continua la innovación, así como su intensidad y la competitividad de acuerdo con las exigencias del mercado global en el momento de competir en un entorno dinámico (Cornett et al., 2019; Jácome y Albán, 2017).

La intensidad de innovación fue moderada-alta y en esta se resalta el modelo de negocio, seguido de productos y mercados. En consecuencia, las empresas orientadas a la exportación del sector textil están obligadas a intensificar sus innovaciones de manera transversal y a todo nivel, lo cual significa que es un proceso iterativo de imaginación, creatividad, invención, entrega y aceptación de la «creación de valor» que genere riqueza y bienestar en las mypes exportadoras del sector textil por la adecuada gestión para sobrevivir, permanecer e incrementar la productividad, especialmente en el objetivo de alcanzar mejores niveles de competitividad con el propósito de contribuir al desarrollo y la calidad de vida en la región y en el país.

Finalmente, la competitividad de las mypes de artesanía textil de la región de Puno, en su mayoría, fue moderada y alta en las dimensiones de productividad, calidad del producto y desempeño en el mercado. La productividad local se convierte en el elemento clave para la competitividad, de modo que da lugar a la ampliación de horizontes para el desarrollo de la economía, al tiempo que conduce al mejor uso de los recursos y a obtener mejores resultados.

Para esto será imprescindible resaltar la calidad de productos con base en la estandarización, el control de calidad y los diseños exclusivos de producto. Procesos que favorecen la generación de una ventaja diferencial a las pymes exportadores de artesanía textil en el mercado internacional y competitivo, y que deben dar lugar a convertirse en políticas públicas por ser de interés nacional, regional y local. 


\section{Limitaciones y futuras líneas de investigación}

$\mathrm{U}$ na de las limitaciones fue que los resultados no se pueden generalizar a todos los sectores de las mypes, debido a que las características del sector son genuinas en el altiplano peruano. No obstante, la investigación puede servir como referentes para aplicar a nivel nacional. Otra limitación del estudio es la explicación de la competitividad solo con la variable innovación.

En las investigaciones futuras es posible analizar estas relaciones con otras variables tales como el conocimiento, el liderazgo, los actores clave de fibra de alpaca y la capacidad de innovación. Por otro lado, se podría utilizar un análisis de ecuaciones estructurales para la determinación de las relaciones de las diferentes proposiciones presentadas.

\section{Referencias}

Akıncı, G.; Utlu, Z. (2015). The research \& development and innovation capacity of Small and Medium Sized Enterprises in IMES. Procedia-Social and Behavioral Sciences, 195, 790-798. DOI: https://doi.org/10.1016/j.sbspro.2015.06.177

Álvarez-Falcón, C. (2017). Innovación, competitividad y nuevos modelos de negocio. Sinergia e Innovación, 1(108). Recuperado de https://bit.ly/3vacKXQ

Aragón, A.; Rubio, A. (2005). Factores explicativos del éxito competitivo: el caso de las pymes del estado de Veracruz. Contaduría y Administración, (216), 35-69. DOI: https://doi. org/10.22201/fca.24488410e.2005.568

Arellano-Cepeda, O.; Quispe-Fernández, G.; Ayaviri-Nina, D.; Escobar-Mamani, F. (2017). Estudio de la aplicación del método de costos $\mathrm{ABC}$ en las mypes del Ecuador. Revista Investigaciones Altoandinas, 19(1), 33-46. DOI: https://doi. org/10.18271/ria.2017.253

Arias, J.; Durango, C. M.; Millán, N. T. (2015). Capacidad de innovación de proceso y desempeño innovador: efecto mediador de la capacidad de innovación de producto. $A D$ Minister, (27), 75-93. DOI: https://doi.org/10.17230/administer.27.4

Arroyo-Cañada, F. J.; Sánchez-Torres, J.; Solé-Moro, M.L. (2017). La calidad e innovación como factores de diferenciación para el comercio electrónico de ropa interior de una marca latinoamericana en España. Contabilidad y Negocios, 12(23), 52-61. DOI: https://doi.org/10.18800/ contabilidad.201701.004

Ascón-Villa, J. E. E.; Espinosa-Manfugás, J. M.; Isla-Ramírez, M.(2018). La situación competitiva de un hotel. Revista Científica Ecociencia, 5(1), 1-30. DOI: https://doi. org/10.21855/ecociencia.51.11
Astudillo, S.; Briozzo, A. (2016). Innovación en las mipymes manufactureras de Ecuador y Argentina. Semestre Económico, 19(40), 117-144. DOI: https://doi.org/10.22395/seec. v19n40a5

Becerra, A. M.; García, E. (2008). Planeamiento estratégico para micro y pequeñas empresas. Lima: Universidad del Pacifico.

Benešová, D.; Kubičková, V.; Michálková, A.; Krošláková, M. (2018). Innovation activities of gazelles in business services as a factor of sustainable growth in the Slovak Republic. Entrepreneurship and Sustainability Issues, 5(3), 452-466. DOI: https://doi.org/10.9770/jesi.2018.5.3(3)

Bezpalov, V. V.; Fedyunin, D. V.; Solopova, N. A.; Avtonomova, S. A.; Lochan, S. A. (2019). A model for managing the innovation-driven development of a regional industrial complex. Entrepreneurship and Sustainability Issues, 6(4), 1884-1896. DOI: https://doi.org/10.9770/jesi.2019.6.4(24)

Cabrera, M.A.; López,P.A.; Ramírez, C. (2011).La competitividad empresarial: un marco conceptual para su estudio. Documentos de Investigación. Administración de Empresas. Bogotá: Ediciones Universidad Central. Recuperado de https://bit. ly/3tLt54V

Camacho, C. K.; Hernández, S. G.; Mayorga, P. I. (2016). La innovación y su interrelación con la competitividad. Sector manufacturero de la zona metropolitana de Guadalajara. Red Internacional de Investigadores en Competitividad, 8(1), 440459. Recuperado de https://bit.ly/3tJzygR

Catacora, M. A. (2013). Modelo de gestión para la competitividad de mypes de la Región Puno (Tesis de doctorado). Universidad Nacional del Altiplano. Puno, Perú. Recuperado de https:// bit.ly/3sLqFSu 
Centro de Estudios Estratégicos-IPAE. (2011). Informe: Compendio notas técnicas 2011. Perú: IPAE Acción empresarial. Recuperado de https://bit.ly/3xhbZ0S

Córdova, I. (2013). El proyecto de investigación cuantitativa. Lima: San Marcos.

Cornett, M. M.; Erhemjamts, O.; Tehranian, H. (2019). Competitive environment and innovation intensity. Global Finance Journal, 41, 44-59. DOI: https://doi.org/10.1016/j. gf. 2019.02.002

Coronado-Medina, A.; Echeverri, A.; Arias-Pérez, J. E. (2015). Aproximación a la cooperación en innovación en empresas del programa de asociatividad y desarrollo empresarial sectorial -PADES- en Antioquia (Colombia). Revista Facultad de Ciencias Económicas, 22(2), 185-205. DOI: https://doi. org $/ 10.18359 /$ rfce. 633

Correa, A. (2005). Intensidad innovadora, permanencia exportadora, y destino de las exportaciones: análisis para una muestra de empresas de Cali. Economía, Gestión y Desarrollo, (3), 9-20. Recuperado de https://bit.ly/32GK4cF

Crittenden, V.L.; Crittenden, W. F.; Ajjan, H. (2019). Empowering women micro-entrepreneurs in emerging economies: The role of information communications technology. Journal of Business Research, 98, 191-203. DOI: https://doi.org/10.1016/j. jbusres.2019.01.045

D'Alessio, F. (2015). Competitividad y productividad para el 2015. Revista Strategia, (35), 14-15. Recuperado de https:// bit.ly/3axH86w

D’Alessio, F.; Del Carpio, L. (2011). Productividad y Competitividad. Revista Strategia, (24), 49-53. Recuperado de https://bit.ly/3tLtog5

Distanont, A.; Khongmalai, O. (2018). The role of innovation in creating a competitive advantage. Kasetsart Journal of Social Sciences, 40(1), 15-21. DOI: https://doi.org/10.1016/j. kjss.2018.07.009

Duarte, R.; Uribe, J. (2012). Desarrollo e innovación artesanal en el Perú: un estudio de caso en Chullucanas, Piura. Revista de Investigación en Ciencias y Administración, 7(12), 193-229.

Dziallas, M.; Blind, K. (2019). Innovation indicators throughout the innovation process: an extensive literature analysis. Technovation, 80-81, 3-29. DOI: https://doi.org/10.1016/j. technovation.2018.05.005

Franco-Ángel, M.; Urbano, D. (2019). Caracterización de las pymes colombianas y de sus fundadores: un análisis desde dos regiones del país. Estudios Gerenciales, 35(150), 81-91. DOI: https://doi.org/DOI:10.18046/j.estger.2019.150.2968

Gálvez, J. E.; García, D. (2012). Impacto de la innovación sobre el rendimiento de la mipyme: un estudio empírico en Colombia. Estudios Gerenciales, 28(122), 11-27. DOI: https://doi. org/10.1016/S0123-5923(12)70191-2
García-Pérez, D.; Gálvez-Albarracín; E. J.; Maldonado-Guzmán, G. (2016). Efecto de la innovación en el crecimiento y el desempeño de las mipymes de la Alianza del Pacífico. Un estudio empírico. Estudios Gerenciales, 32(141), 326-335. DOI: https://doi.org/10.1016/j.estger.2016.07.003

Ghosh, A.; Kato, T.; Morita, H. (2017). Incremental innovation and competitive pressure in the presence of discrete innovation. Journal of Economic Behavior and Organization, (135), 1-14. DOI: https://doi.org/10.1016/j.jebo.2016.12.022

Girón, A. (2016). Objetivos del desarrollo sostenible y la Agenda 2030: frente a las políticas públicas y los cambios de gobierno en América Latina. Problemas del Desarrollo, 47(186), 3-8. DOI: https://doi.org/10.1016/j.rpd.2016.08.001

Gonzales, S.; Gonzáles, D. (2017). Innovación en un modelo de negocio con impacto en la gestión de la calidad. Caso gran empresa de soluciones eléctricas integrales. Ponencia presentada en el XVII Congreso Latino-Iberoamericano de Gestión Tecnológica ALTEC 2017. Asociación Latino-Iberoamericana de Gestión Tecnológica (ALTEC), Ciudad de México, México, 16-18 de octubre. Recuperado de https://bit.ly/3aPnLGv

Guevara, A. (2006). Espejismos desarrollistas y autonomía comunal: el impacto de los proyectos de desarrollo en el lago Titicaca (1930-2006). Derecho PUCP, (62), 319-320. Recuperado de https://bit.ly/3vfFBd8

Heredia-Rodríguez, L.; Sánchez-Macías, J. I. (2016). Evolución de las políticas públicas de fomento a las pymes en la Comunidad Andina de Naciones y la Unión Europea: un análisis comparativo. Finanzas y Política Económica, 8(2), 221-249. DOI: https://doi.org/http://dx.doi.org/10.14718/ revfinanzpolitecon.2016.8.2.2

Hitt, M. A.; Ireland, R. D.; Hoskisson, R. E. (2015). Administración estratégica: competitividad y globalización: conceptos y casos. México, D. F.: Centage Learning Editores.

Instituto Nacional de Estadística e Informática. (2015). Perú: Encuesta nacional de innovación en la industria manofacturera. Lima: INEI. Recuperado de https://bit.ly/2QQgRJS

Instituto Nacional de Estadística e Informática. (2016). Perú: Estructura Empresarial. Lima: INEI. Recuperado de https:// bit.ly/3aAthwe

Jácome, I.; Albán, M. (2017). La innovación y el nuevo producto en desarrollo. Revista de Ciencias Humanísticas y Sociales, (ES),117-125. DOI: https://doi.org/10.33936/rehuso. v0i0.1005

Jasso,J.(1998).Desempeño innovadory competitividad internacional. México D.F.: Centro de Investigación y Docencia Económica.

Kang, T.; Baek, C.; Lee, J. D. (2019). Effects of knowledge accumulation strategies through experience and experimentation on firm growth. Technological Forecasting and Social Change, (144), 169-181. DOI: https://doi. org/10.1016/j.techfore.2019.04.003 
Karabulut, A. T. (2015). Effects of innovation types on performance of manufacturing firms in Turkey. Procedia-Social and Behavioral Sciences, 195, 1355-1364. DOI: https://doi. org/10.1016/j.sbspro.2015.06.322

Khan, K. U.; Xuehe, Z.; Atlas, F.; Khan, F. (2018). The impact of dominant logic and competitive intensity on SMEs performance: A case from China. Journal of Innovation \& Knowledge, 4(1), 1-11. DOI: https://doi.org/10.1016/j. jik.2018.10.001

Lewin, A. Y.; Välikangas, L.; Chen, J. (2017). Enabling open innovation: lessons from Haier. International Journal of Innovation Studies, 1(1), 5-19. DOI: https://doi.org/10.3724/ sp.j.1440.101002

Maldonado, G. (2011). La influencia de las TIC y la innovación en el rendimiento de las pymes de Aguascalientes: una evidencia empírica. Mercados y Negocios: Revista de Investigación y Análisis, 12(1), 15-34.

Martínez-Marín, M. I.; Santero-Sánchez, R.; Sánchez-Henríquez, L.; Marcos-Calvo, M. Á. (2009). Factores de la competitividad de la pyme española 2008. Madrid: Fundación Escuela de Organización Industrial. Recuperado de https://bit. ly/3avsBbw

Mendoza, J. M. (2010). La capacidad competitiva. Bogotá: Corporación para la Gestión del Conocimiento ASD 2000.

Ministerio de Comercio Exterior y Turismo. (2015). PENX Plan estratégico nacional exportado 2025. Lima: MINCETUR. Recuperado de https://bit.ly/3xfH9WD

Monzón, A.; Hernández-Pérez, G. D.; Nogueira-Rivera, D. (2015). Intensidad innovadora en empresas de base tecnológica en el sector hidráulico cubano. Ingeniería Industrial, XXXVI(1), 82-90.

Moraes, D.; Buesa, M.; Johannes, J.; Baumert, T. (2018). Innovación y competitividad: un análisis aplicado a las empresas industriales españolas. Cuadernos de Información Económica, (265), 25-41.

Morales, M.; León, A. (2013). Adiós a los mitos de la innovación. Una guía práctica para innovar en América Latina. Innovare.

Mora-Riapira, E. H.; Vera-Colina, M. A.; Melgarejo-Molina, Z. A. (2015). Planificación estratégica y niveles de competitividad de las mipymes del sector comercio en Bogotá. Estudios Gerenciales, 31(134), 79-87. DOI: https://doi.org/10.1016/j. estger.2014.08.001

Münch, L. (2007). Administración: escuelas, proceso administrativo, áreas funcionales y desarrollo emprendedor. México, D. F.: Pearson.

Orengo-Serra, K. L.; Ortíz-Soto, M. (2017). Factores que el fabricante/distribuidor toma en consideración para la comercialización de alimentos especiales en mercados foráneos. Estudios Gerenciales, 33(144), 281-291. DOI: https://doi.org/DOI:10.1016/j.estger.2017.06.003
Osterwalder, A.; Pigneur, Y. (2011). Generación de modelos de negocio: un manual para visionarios, revolucionarios y retadores. Barcelona: Deusto.

Paredes, B. G. (2017). Análisis de la competitividad empresarial $y$ su incidencia en el mercado internacional del consorcio textil Titicaca Puno, periodo 2014-2015 (Tesis de grado). Universidad Nacional del Altiplano. Puno, Perú.

Pat, V. G.; Caamal, I.; Ávila, J. A. (2009). Análisis de los niveles y enfoques de la competitividad. Textual, (53), 63-76.

Porter, M. E. (1990). The competitive advantage of nations. Harvard Business Review. DOI: https://doi. org/10.1007/978-1-349-11336-1

Rajapathirana, R. P. J.; Hui, Y. (2018). Relationship between innovation capability, innovation type, and firm performance. Journal of Innovation \& Knowledge, 3(1), 4455. DOI: https://doi.org/10.1016/j.jik.2017.06.002

Reshetnikova, N. N.; Magomedov, M. G. (2020). Influence strategic competitive advantage international business cooperation in the frame of financial crisis. Springer Nature Switzerland, 138, 399-408. DOI: https://doi. org/10.1007/978-3-030-15577-3_39

Ríos-Manríquez, M. (2017). Ethical behavior of small enterprises. An empirical case. Holos, 5, 158-174. DOI: https://doi.org/10.15628/holos.2017.4499

Rodeiro-Pazos, D.; Simbaña-Taipe, L. E.; Rodríguez-Gulías, M. J.; Fernández-López, S. (2018). Effects of innovation on the growth of ecuadorian firms: a quantile analysis. Journal of Business, 10(2), 70-87. DOI: https://doi.org/10.21678/ jb.2018.851

Salunke, S.; Weerawardena, J.; McColl-Kennedy, J. R. (2019). The central role of knowledge integration capability in service innovation-based competitive strategy. Industrial Marketing Management, 76, 144-156. DOI: https://doi. org/10.1016/j.indmarman.2018.07.004

Sawhney, M.; Wolcott, R. C.; Arroniz, I. (2007). Las doce formas de innovar para las empresas. Harvard Deusto Business Review, (153), 22-32.

Shafi, M.; Yang, Y.; Khan, Z.; Yu, A. (2019). Vertical cooperation in creative micro-enterprises: a case study of textile crafts of Matiari District, Pakistan. Sustainability, 11(3), 20-23. DOI: https://doi.org/10.3390/su11030920

Suarez, R. M.; Rodríguez, Y. A.; Muñoz, N. (2017). Análisis de percepción sobre estrategias administrativas y el impacto en la productividad laboral. Revista Ingeniería, Matemáticas y Ciencias de la Información, 4(8). 61-67. DOI: http://dx.doi. org/10.21017/rimci.2017.v4.n8.a33

Tavera, J. (2014). El estado de la innovación tecnológica en el Perú. Revista de Economía San Marcos, 1(1), 41-51. 
Tohidi, H.; Jabbari, M. M. (2012). Important factors in determination of innovation type. Procedia Technology, 1, 570-573. DOI: https://doi.org/10.1016/j. protcy.2012.02.124

Trías, F.; Kotler, P. (2011). Innovar para ganar. Barcelona: Ediciones Urano.

Valdés, L. (2009). El dado de 7 caras: guía e inspiración para encontrar oportunidades innovadoras de negocio. Barcelona: Plataforma.

Valencia-Durand, R. A.; Duche-Pérez, A. B. (2019). Innovación de la gestión y éxito competitivo en medianas y grandes empresas del sector manufacturero peruano. Universidad y Sociedad, 11(4), 141-153. Recuperado de https://bit. ly/2PeMZX9

Verreynne, M. L.; Williams, A. M.; Ritchie, B. W.; Gronum, S.; Betts, K. S. (2019). Innovation diversity and uncertainty in small and medium sized tourism firms. Tourism Management, 72, 257-269. https://doi.org/10.1016/j. tourman.2018.11.019

Yarce, J. (2000). Los valores son una ventaja competitiva: cómo aprender a practicarlos personalmente: cómo construir una organización basada en valores. Bogotá: Ediciones ILL.

Yu, L.; Duan, Y.; Fan, T. (2020). Innovation performance of new products in China's high-technology industry. International Journal of Production Economics, 219, 204-215. DOI: https://doi.org/10.1016/j.ijpe.2019.06.002 